ARTIGO DE REVISÃO REVIEW ARTICLE

\section{Diferentes metodologias para a avaliação de políticas em saúde mental no Brasil e no mundo: uma revisão teórica}

\author{
Distinct methodologies for mental health policy evaluation \\ in Brazil and the world: a theoretical review \\ Pedro Henrique de Morais Campetti ${ }^{1}$
}

DOI: $10.21115 / J B E S . v 11 . n 3 . p 263-70$

\begin{abstract}
RESUMO
Transtornos mentais como a depressão, a ansiedade e a distimia são enfermidades que afetam gravemente a população mundial e são uma das principais causas de incapacidade no trabalho tanto no Brasil quanto em diversos outros países. Estima-se que até 36 milhões de pessoas sofram de depressão no país e até 350 milhões ao redor do mundo. Trata-se de um problema econômico, social e humano, pois provoca diminuição do bem-estar dos indivíduos e de toda a sociedade, ao reduzir a renda e a riqueza, a felicidade e as oportunidades reais das pessoas de fazer e ser o que valorizaram. É nesse contexto que a Economia possibilita ferramentas para análise de políticas públicas, neste caso, aquelas relacionadas à saúde mental. Para tanto, o procedimento metodológico utilizado foi uma revisão de natureza teórica sobre os diferentes métodos que podem ser empregados para a avaliação da saúde mental no Brasil e no mundo. Foram descritos três referenciais teóricos: a Economia Tradicional, a Economia da Felicidade e a Abordagem das Capacitações. Os resultados apontam que cada uma dessas três abordagens econômicas permite entender, de um ângulo diferente, os problemas relacionados às doenças mentais, enriquecendo, assim, as possibilidades de promoção de políticas públicas. Entretanto, ainda são poucos os estudos sobre o tema em Economia da Felicidade e na Abordagem das Capacitações. No Brasil, tais estudos concernentes à saúde mental são exíguos, independentemente da metodologia utilizada.
\end{abstract}

\section{Keywords:}

public policies, public health, economic assessment, mental disorders, depression

\begin{abstract}
Mental disorders such as depression, anxiety and dysthymia are diseases that severely affect the world's population and are a major cause of disability at work both in Brazil and in many other countries. It is estimated that up to 36 million people suffer from depression in Brazil and up to 350 million around the world. It is an economic, social and human issue because it causes a decrease in the well-being of individuals and the whole of society by reducing income and wealth, happiness and real opportunities for people do and be what they value. In this regard, Economics provides tools for analysis of public policy, in this case those related to mental health. Therefore, the methodological procedure employed is a theoretical review on the different methods that may be employed for mental health assessment in Brazil and worldwide. Three theoretical references were reported: the Traditional Economics, the Happiness Economics and the Capability Approach. The results show that each of these three economic approaches allows to understand the concerns related to mental illnesses from a different angle, thus enriching the possibilities of promoting public policies. However, there are still few studies on the subject of Happiness Economics and, specially, the Capability Approach. In Brazil, such studies concerning mental health are lacking regardless of the methodology used.
\end{abstract}




\section{Introdução}

Doenças como a depressão, a ansiedade e a distimia afetam, respectivamente, 298, 273 e 106 milhões de pessoas, o que representa 4,3\%, 3,7\% e 1,5\% da população mundial. Ademais, a depressão é a 19a doença mais comum na população, enquanto a ansiedade é a 21a (Vos et al., 2012). Outras estimativas apontam que, no ano de 2015, a prevalência mundial de depressão foi de 322 milhões de casos, enquanto a de transtornos de ansiedade foi de 264 milhões de pessoas afetadas (WHO, 2017). Comparativamente a outras nações em desenvolvimento, o Brasil está em primeiro lugar na prevalência de depressão. Constata-se que a doença afeta entre 10\% e 18\% da população no período de um ano, o que equivale entre 20 e 36 milhões de indivíduos ou 6,6\% a 12\% da população com depressão no mundo (Razzouk, 2016b). Apesar disso, em razão dos estigmas, preconceitos e medos relacionados ao tema, os transtornos mentais permanecem como um assunto obscuro.

Nesse contexto, as Ciências Econômicas podem contribuir significativamente no estudo, análise e debate das questões envolvendo a saúde das populações, o que inclui as doenças mentais. Um dos principais objetos de estudo da Economia é a avaliação do bem-estar das sociedades e a promoção do desenvolvimento humano. Nesse contexto, três são as principais referências em uso na atualidade: a Economia Tradicional ou Mainstream, a Economia da Felicidade e a Abordagem das Capacitações. Cada uma delas enfatiza os aspectos que mais importam para o bem-estar e o progresso econômico e social (Stiglitz et al., 2009). Ou seja, podem-se utilizar os três referenciais para uma avaliação abrangente e multidimensional de determinado assunto: A Economia Tradicional possui em seu bojo teórico a análise de custo-utilidade, que é considerada uma das formas mais amplamente utilizadas de avaliação econômica, podendo ser empregada na área da saúde. O objetivo, nesse caso, é o da maximização da utilidade em relação aos custos (Hunt, 2005; Luyten et al., 2016). Por sua vez, a Economia da Felicidade valoriza o bem-estar subjetivo, isto é, a percepção dos indivíduos quanto a sua própria vida. Assim, seu foco de estudo é o entendimento quanto aos principais fatores de felicidade ou infelicidade, sendo a meta promover a maior felicidade possível da população (Mcgillivray, 2007). Por último, a Abordagem das Capacitações destaca as liberdades substantivas das pessoas para ser, fazer e viver uma vida que valha a pena. Nesse caso, pessoas com problemas de saúde podem, muitas vezes, ter suas liberdades limitadas (Mcgillivray, 2007).

Nota-se que os transtornos mentais são uma questão de saúde pública. O impacto deles pode resultar em três diferentes problemas - que serão detalhados nas próximas seções -, cada um deles relacionado aos três referenciais teóricos apresentados anteriormente: (1) a redução da renda e da produtividade na sociedade e o aumento dos custos com saúde; (2) a diminuição da felicidade média da população e uma das principais causas de miséria em termos de bem-estar subjetivo; e (3) as limitações de agência dos indivíduos de levarem uma vida plena. Nesse sentido, o objetivo desta pesquisa é fazer uma revisão de natureza teórica - tendo por objeto de estudo a depressão e os transtornos de ansiedade - sobre a avaliação da saúde mental no Brasil e no mundo com um enfoque multidimensional. Mais especificamente, trata-se de uma descrição de três abordagens para avaliação de políticas públicas e exemplos de sua aplicação na saúde, especialmente quanto aos problemas de saúde mental. Para tanto, são desenvolvidos três ensaios, cada qual abordando um dos referenciais econômicos apresentados.

Desse modo, nas seções seguintes é detalhada cada uma dessas abordagens e a relação com a saúde mental. Por último, há as considerações finais, na qual são abordados os benefícios de uma análise ampla e multidimensional sobre o assunto em estudo e os desafios para as pesquisas futuras.

\section{Análise de custo-benefício em saúde mental}

Um conceito basilar em Economia é que os recursos são escassos e o problema econômico fundamental está em escoIher a alocação ótima. Nesse contexto, qualquer sistema de saúde se depara com recursos limitados e o princípio orientador está em decidir como direcioná-los para que alcancem o maior benefício possível.

Esse exercício pode ser feito por meio de uma avaliação econômica em saúde, havendo diferentes metodologias para isso. Em primeiro lugar, é possível fazer uma verificação que leva em conta apenas os custos das doenças e das intervenções. Nesse caso, como a avaliação está restrita aos custos, o objetivo está em minimizá-los. Isso significa que não há a perspectiva da eficiência, isto é, não se considera o valor adicionado para o investimento feito. Por exemplo, pode-se estimar qual o custo médio de um paciente para o sistema de saúde ou para a sociedade. Por outro lado, uma análise abrangente leva em conta os efeitos de duas ou mais opções de intervenção. Há três métodos para fazer isso: (1) análise de custo-efetividade; (2) análise de custo-utilidade; e (3) análise de custo-benefício. Essas são idênticas em suas formulações sobre como capturar custos, diferindo, porém, em como avaliam os efeitos na saúde. Em resumo, a primeira compara os custos econômicos e os efeitos à saúde de duas ou mais intervenções; na segunda, as consequências em saúde são traduzidas em uma medida genérica de saúde (por exemplo, anos de vida ganho, ajustados pela qualidade - QALY em inglês - ou anos de vida corrigidos pela incapacidade - DALY em inglês); e no terceiro caso, o resultado é mensurado em unidades monetárias (Luyten et al., 2016; Silva et al., 2016). Diversos estudos têm analisado a incidência e os custos com enfermidades mentais, os gastos médios em tratamento e os retornos obtidos de acordo com o investimento, os quais são discutidos a seguir. 
Primeiramente, importa distinguir que os custos com problemas de saúde mental podem ser classificados como diretos, correspondentes aos gastos com tratamento e assistência ao paciente, ou indiretos, relacionados a questões como redução da produtividade, morte prematura, uso de benefícios sociais diversos, menor escolaridade, entre outros (Razzouk, 2016a). Entre os custos indiretos, há aqueles correspondentes ao ambiente laboral; são eles: absenteísmo, presenteísmo e aposentadoria precoce. Absenteísmo refere-se ao afastamento do trabalho, no caso em questão por motivo de doença mental. Presenteísmo é um neologismo que designa uma menor produtividade, pois a pessoa está apenas de "corpo presente", mas sem desempenhar bem suas funções. Nesses casos, o custo é representado pela redução da produtividade, que, de acordo com um estudo da OECD (2012), chega a ser 6\% menor em razão desses fatores.

Um estudo de Chisholm et al. (2016) chegou ao resultado de uma perda de produtividade, atribuída tanto ao absentismo quanto ao presenteísmo, na ordem de 12 bilhões de dias por ano em razão de transtornos de depressão e ansiedade nos 36 maiores países do mundo. Isso equivale a um custo estimado de 925 bilhões de dólares ou em 1,15 trilhão de dólares ao se projetarem os dados para o mundo todo.

Outras publicações tentam estimar o tamanho do custo do absenteísmo, fazendo algumas comparações. Uma pesquisa no Reino Unido demonstrou que o custo com depressão de adultos era estimado em 9 bilhões de libras, e apenas 370 milhões (ou 4\%) representavam os custos diretos com tratamento (Thomas \& Morris, 2003). Já nos Estados Unidos, um estudo mostrou que 62\% dos custos com depressão estavam relacionados ao absenteísmo, presenteísmo e desemprego, 7\% eram devidos à mortalidade culminada pelo suicídio e 31\% eram referentes aos custos médicos diretos (Greenberg et al., 2003). Em outro estudo, os dados para 374.799 empregados de seis grandes empresas foram analisados. As perdas por incapacidade constituíram 29\% do total de gastos relacionados à saúde e à produtividade para condições de saúde física e 47\% para todas as condições de saúde mental examinadas (Goetzel et al., 2003). Em relação ao afastamento do trabalho no Brasil, a depressão é considerada o terceiro principal fator demandante de auxílio doença no Instituto Nacional do Seguro Social - INSS (Razzouk, 2016a).

Em relação aos gastos com saúde mental, verifica-se que há uma considerável disparidade entre os países de alta renda e países de baixa renda. Nestes, a despesa per capita é de 0,20 dólar americano, enquanto naqueles é de 44,84 dólares, uma diferença de mais de 200 vezes. Nos países de baixa-média e de alta-média renda, os gastos com saúde mental são de 0,59 e 3,76 dólares, respectivamente, e a média global fica em 1,63 dólar. Também há discrepâncias quando se averigua o percentual médio do orçamento da saúde destinado à saúde mental por grupo de renda: os países de baixa, média-baixa, média-alta e alta renda investem o equivalente a 0,53\%, 1,90\%, 2,38\% e 5,10\% do seu orçamento (WHO, 2011).

Nas pesquisas apresentadas, pode-se observar que os custos indiretos são mais representativos que os custos diretos. Ou seja, não são os gastos com tratamentos médicos, farmacológicos e psicológicos o direcionador mais importante dos custos totais da doença, mas sim os custos laborais e sociais dessa doença. Por exemplo, além dos custos apresentados anteriormente, também há os relacionados à mortalidade. Segundo Mykletun et al. (2009), a taxa de risco (hazard ratio) da associação entre depressão e mortalidade é equivalente a 1,52, algo um pouco inferior ao uso de cigarros, em que, em comparação, o risco relativo é de 1,59. Em outro estudo feito por meio de uma metanálise de dados de 54 pesquisas, Nicholson et al. (2006) buscaram quantificar o efeito da depressão na etiologia e prognóstico das doenças do coração. Eles averiguaram que o risco relativo (relative risk) de um indivíduo vir a ter uma doença do coração associada à depressão ficou entre 1,53 e 1,90, dependendo do grupo de estudos utilizado e da doença cardíaca.

Apesar da importância dos dados discutidos anteriormente, uma análise completa deve levar em conta os efeitos de uma determinada intervenção. Nesse caso, procura-se entender os possíveis cursos de ação, seus custos e também seus resultados, de modo que possam ser comparados.

Para verificar os resultados do tratamento, Laynard et al. (2007) fizeram uma análise de custo-benefício do fornecimento de psicoterapia para pessoas com depressão ou transtorno de ansiedade no Reino Unido. Eles verificaram que o custo médio do tratamento por meio de terapia é pequeno, equivalente a 750 libras, sendo a taxa de recuperação equivalente a 50\%. Por outro lado, os gastos do governo por pessoa com assistência foram calculados em 9.000 libras por ano ou 750 por mês. Os autores também estimaram os ganhos totais para a sociedade: 4.700 libras em dois anos com o tratamento da depressão. Esse valor é resultado da soma de três fatores: (1) o produto interno bruto (PIB) produzido pelo tratamento de uma pessoa é de 1.100 libras em razão dos ganhos extras de 1,1 mês a mais de trabalho; (2) a economia com despesas do Serviço Nacional de Saúde é de 300 libras; e (3) o ganho relativo aos anos de vida ajustados pela qualidade (QALYs) é equivalente a 3.300 libras. Esse último valor é resultado da multiplicação entre o ganho esperado de 0,11 QALYs por pessoa tratada por 30.000 libras, sendo esse o valor-padrão ou limiar aceitável de custo-efetividade do National Institute for Health and Care Excellence (NICE).

Em relação a este último, os autores consideraram o ganho esperado em vida saudável por pessoa tratada de 0,55 ano nos dois anos subsequentes, o que é igual a. Ao fazer a equivalência de 1 QALY igual a 30.000 libras, então 0,11 QALY é igual a 3.300 libras.

No Brasil, as pesquisas que avaliam os transtornos mentais em termos de custo-benefício costumam relacionar como 
essas doenças ocasionam o absenteísmo no trabalho para uma determinada região (Cruz et al., 2011; Faria et al., 2005; Fonseca \& Carlotto, 2011; Pawlina al., 2009; Santana et al., 2016; Santos \& Mattos, 2010; Silva et al., 2012; Silva Junior \& Fischer, 2015). Por outro lado, são ainda incipientes as avaliações relacionadas aos custos (nas suas várias óticas) do adoecimento mental (Razzouk, 2013; Silva-Junior \& Fischer, 2014), e não foi encontrado nenhum estudo que estime, para o país, o ganho ou os benefícios (por exemplo, em produtividade) com tratamento ou o impacto de políticas públicas na área.

\section{O impacto das doenças mentais na felicidade}

Uma segunda forma de avaliar questões relacionadas à saúde é verificando o impacto de diversas doenças no bem-estar subjetivo das pessoas. Essa perspectiva, em Economia, tem início com Richard Easterlin (1974) e ficou conhecida como Economia da Felicidade. Desde então, estudos têm buscado entender quais são os determinantes da felicidade dos indivíduos e a relação disso com as políticas públicas. Verificou-se que diversas variáveis importam para a felicidade, por exemplo, a situação financeira, relacionamentos familiares, trabalho, comunidade e amigos, liberdade pessoal, valores pessoais, características sociodemográficas e saúde (Layard, 2008; Frey \& Stutzer, 2002). Em relação à última variável - saúde -, podem-se avaliar aspectos específicos, como a implicação de problemas de depressão e ansiedade para a felicidade das pessoas e para o índice de felicidade da população. Também é possível buscar as causas, fatores ou características populacionais associadas às doenças mentais.

Em primeiro lugar, vale ressaltar que a saúde é um dos principais ingredientes da felicidade e há uma elevada correlação entre essas duas variáveis (Easterlin, 2005; Frey \& Stutzer, 2002; Layard, 2008; Mehnert et al., 1990). Um exemplo de estudo empírico foi o desenvolvido por Campetti e Alves (2015) para seis países da América Latina, que teve por objetivo verificar os determinantes da felicidade dos indivíduos, sendo a saúde um dos principais fatores. No seu modelo de regressão, os autores observaram que essa variável é estatisticamente significante e possui elevada correlação com a felicidade. Além disso, pessoas mais saudáveis eram, em média, 0,9 ponto mais felizes que pessoas com estado de saúde regular ou ruim. Entretanto, alguns autores como Frey e Stutzer (2002) e Layard (2008) declaram que, em muitas circunstâncias, as pessoas se adaptam às suas condições de saúde, retornando ao mesmo nível de felicidade. Um exemplo são as pessoas que ficam paraplégicas: transcorrido algum tempo do incidente que ocasionou a perda dos movimentos dos membros inferiores, elas tendem a se ajustar à situação. Além disso, quanto menos grave a condição de saúde da pessoa, mais provável é que ela se adapte. Por outro lado, há casos em que a adaptação não acontece ou não é completa. Frederick e Loewenstein (1999) destacam que as doenças mentais como a depressão se enquadram nessa situação, pois são relativas à subjetividade e aos fatores internos do próprio indivíduo.

Em segundo lugar, pode-se destacar especificamente o papel da saúde ou das doenças mentais para a felicidade ou infelicidade dos indivíduos. No trabalho de Clark et al. (2017) foi analisado o quanto a miséria - entendida em Economia da Felicidade quando uma pessoa está abaixo de um valor de corte em relação a sua satisfação com a vida como um todo - seria reduzida se fosse possível eliminar a pobreza, a baixa escolaridade, o desemprego, a situação de viver sozinho e/ou uma doença física e/ou uma mental. Os autores constaram que, para os quatro países pesquisados (Estados Unidos, Austrália, Reino Unido e Indonésia), a eliminação dos transtornos como depressão e ansiedade, que estão entre as principais formas de doença mental, teria o maior efeito sobre a redução da miséria. Mais que isso, exceto para a Indonésia, a saúde mental explica mais a variação da felicidade do que a renda. Em nenhum lugar a doença física é uma fonte maior de miséria do que a doença mental. Por exemplo, nos Estados Unidos, uma pessoa que é pobre tem 5,5\% a mais de probabilidade de ser infeliz, enquanto alguém com depressão ou ansiedade tem uma propensão de 10,7\%. Layard et al. (2013) encontrou o mesmo resultado para o Reino Unido, a Alemanha e a Austrália, nos quais os problemas de saúde mental representam a variável explicativa mais importante para a felicidade dos indivíduos (coeficientes iguais a 0,46, 0,26 e 0,28, respectivamente, para cada um dos países citados e estatisticamente significante a $1 \%$ ), superando as doenças físicas (coeficientes de 0,08, 0,16 e 0,08 e estatisticamente significante a 1\%), que, por sua vez, são mais importantes que a renda ou o desemprego.

Segundo Kim-Cohen et al. (2003), entre os casos de adultos diagnosticados com algum problema psiquiátrico, 73,9\% haviam recebido o diagnóstico antes dos 18 anos de idade e 50,0\%, antes dos 15 anos de idade. Clark et al. (2017) corroboram ao afirmar que o mais forte indicador de uma vida adulta satisfatória não está nas qualificações adquiridas por meio da educação formal, mas numa combinação entre comportamento e saúde emocional da criança, com destaque para esta última variável. Outros estudos como o de Layard et al. (2012) e o de Layard (2014) apontam que, em pesquisa para avaliar o bem-estar de homens britânicos de 34 anos, a variável explicativa mais poderosa foi o "mal-estar" - que representa a saúde mental por meio de 24 perguntas - reportado oito anos antes, superando outras variáveis como a saúde geral oito anos antes e a renda atual. E mesmo quando a saúde mental seja medida aos 16 anos, ela ainda exerce quase tanto impacto na satisfação com a vida aos 34 anos quanto a renda atual.

Quanto ao efeito dos pais sobre o desenvolvimento infantil, Clark et al. (2017) verificaram que a renda familiar tem efeito substancial no desempenho acadêmico, mas efeito muito menor na saúde emocional e no comportamento da criança. Nesse caso, o que mais importa em uma série de 
variáveis analisadas é a saúde mental da mãe, que possui correlação com a saúde mental do(s) filho(s).

Portanto, tais estudos fornecem algumas pistas sobre a felicidade dos indivíduos, o que causa seu aumento ou redução, e de que modo a política pública pode ser direcionada para o aumento da felicidade geral. Conforme analisado, as doenças mentais como a depressão, a ansiedade e a distimia são fatores preponderantes para a miséria em termos de bem-estar subjetivo. Não só isso, questões relacionadas à saúde mental dos jovens e das crianças impactam seu desenvolvimento adulto. Para chegar a tais conclusões, os economistas têm utilizado o ferramental matemático para analisar quais são as variáveis explicativas de uma determinada questão. Uma das descobertas é que, para uma agenda em prol da felicidade, há a necessidade de maiores gastos em saúde mental. Nesse sentido, Layard et al. (2013) consideram que um melhor tratamento aumentaria diretamente a felicidade e uma maior felicidade reduziria a frequência de doenças mentais. Para um mundo mais feliz, é preciso um acordo completamente novo sobre saúde mental.

\section{As avaliações em saúde e a abordagem das capacitações}

Conforme estudado nas duas seções anteriores, os problemas de saúde mental têm impactos econômico e social pelos seus custos explícitos e implícitos e pela redução da felicidade dos indivíduos. Há, todavia, um terceiro aspecto a ser considerado, tendo por base a Abordagem das Capacitações [The Capability Approach em inglês], que tem entre seus maiores expoentes Amartya Sen e Martha Nussbaum. Nessa perspectiva, uma vida boa não é necessariamente uma vida dita, pelo próprio indivíduo, como sendo feliz ou satisfatória, ou mesmo rica de bens materiais, mas sim uma vida de escolhas genuínas. O que importam são coisas que as pessoas são livres para ser e fazer (Sen, 2008). A saúde e, em especial, a saúde mental têm importância instrumental nessa análise, pois muitas vezes a falta delas, em conjunto com outros atributos socioeconômicos como a pobreza e a exclusão social, limita as liberdades dos indivíduos.

A Abordagem das Capacitações é uma estrutura teórica que envolve dois princípios normativos fundamentais: primeiro, a afirmação de que a liberdade de alcançar o bem-estar é de importância moral primária, e, segundo, que a liberdade para alcançar o bem-estar deve ser entendida em termos de capacitações [capability] dos indivíduos, isto é, as suas oportunidades reais para fazer e ser o que têm motivos para valorizar (Robeyns, 2011 ). Além disso, essa corrente teórica crítica os pressupostos da Economia Tradicional e, mais sutilmente, da Economia da Felicidade. Em relação à primeira, Sen (2010) expõe algumas desvantagens tais como: indiferença distributiva, por não considerar a desigualdade na distribuição das utilidades; descaso com os direitos e liberdades, valorizando estas apenas na proporção em que influenciam a utilidade; e adaptação e condicionamento mental, no sentido de os desejos e prazeres dos indivíduos serem adaptáveis às circunstâncias, sobretudo para tornar a vida suportável diante das adversidades. Em relação à Economia da Felicidade, Sen (2011) crítica o pressuposto da felicidade ser considerada, por autores como Layard (2008), um objetivo último, no qual estaria implícita a afirmação de que, em última instância, nada mais importa. Sen (2011) argumenta que a felicidade não pode ser concebida como sendo a única coisa que as pessoas têm razão para valorizar, nem mesmo a principal métrica de bem-estar, independentemente da importância que ela tenha.

Para Sen (2008; 2010), o bem-estar de um indivíduo deve levar em conta seu conjunto capacitatório, isto é, sua liberdade de escolher entre atividades que ele considera valiosas e estados de ser (suas "funções"). Em outras palavras, o que importa não são especificamente as condições ou os "estados" do indivíduo, mas a liberdade para escolher o que quer ser ou fazer. O exemplo clássico de Sen é o caso de dois indivíduos que estão famintos, mas a diferença é que um escolheu jejuar, enquanto o outro tem essa condição imposta. No caso da saúde, tem-se o status de saúde como a "função", enquanto a "capacitação" está na liberdade em buscar melhorias na saúde. Outra questão é que Sen não fornece uma lista única de capacitações, deixando que essa escolha seja feita por meio da deliberação pública. Por outro lado, Nussbaum (2011) propôs as 10 Capacitações Humanas Centrais, as quais fornecem um conjunto universal de capacitações, sendo algumas intrinsicamente relacionadas à saúde e também à saúde mental.

No contexto da Abordagem das Capacitações, a saúde tem importância fundamental. Ela está relacionada a outras capacitações, influenciando as oportunidades mais amplas que alguém tem na vida, e, de modo inverso, pode ser influenciada por outros componentes como nutrição, fatores ambientais, educação e renda (Simon et al., 2013). Não por acaso, a estrutura das capacitações levou à introdução do Índice de Desenvolvimento Humano (IDH) pelo Programa das Nações Unidas para o Desenvolvimento em 1990, sendo uma das três dimensões a saúde, por meio do indicador expectativa de vida.

Outra questão está relacionada ao uso do indicador QALYS pela economia tradicional. Por um lado, há uma relação entre capacitações e QALY, pois este incorpora expectativa de vida, também utilizada no IDH. Por outro lado, há distintos aspectos da saúde, como mobilidade e dor, que têm implicação tanto para as capacitações quanto para as funções dos indivíduos. Ou seja, tal como a renda, o status de saúde importa, mas é uma medida imperfeita de acordo com a Teoria das Capacitações. Um dos motivos para isso é que não é possível assumir que o bem-estar em outras áreas da vida seja independente do status de saúde. A abordagem das capacitações procura, então, avaliar intervenções que beneficiem tanto a 
saúde quanto outros aspectos da vida humana. Por exemplo, dois indivíduos podem ter o mesmo QALY para o resto de suas vidas após uma intervenção, mas diferentes oportunidades, pois um tem maior dificuldade de achar emprego em razão do seu status de saúde (Anand, 2005).

Em outras palavras, há a necessidade de realizar avaliações econômicas abrangendo resultados tanto em saúde quanto em aspectos não relacionados à saúde. Assim, é possível fornecer uma alternativa à análise de maximização em saúde, em que se comparam os custos por QALY adquirido. A Abordagem das Capacitações de Sen, embora teoricamente desafiadora, poderia fornecer uma solução possível (Lorgelly et al., 2010). Alguns pesquisadores têm encarado esse desafio. Lorimer et al. (2007) e Lorgelly et al. (2008) buscaram desenvolver, em cada uma das pesquisas, um questionário de acordo com a abordagem das capacitações para uso na avaliação de intervenções sociais e de saúde pública. Zaidi e Burchardt (2005) e Kuklys (2005) estimaram a renda adicional necessária para pessoas com deficiência, de modo que alcancem o bem-estar de uma pessoa sem deficiência. Kinghorn et al. (2007) desenvolveram uma avaliação de qualidade de vida para pessoas que sofrem de dor crônica.

Em relação à saúde mental, Simon et al. (2013) desenvolveram um questionário que operacionaliza a abordagem das capacitações para pesquisas na área. Esse instrumento de coleta de dados foi construído tendo por base teórica a lista das 10 capacitações de Nussbaum (2011) e também o índice de 18 capacitações de Lorgelly et al. (2008), sendo, então, aplicado para 333 pacientes do serviço de saúde mental da Inglaterra. Os resultados apontaram correlação com a utilidade observada em outros questionários de saúde. Também apresentaram as capacitações mais afetadas, que são: "atividades diárias", "influência sobre decisões locais", "aproveitar a recreação", "planejar a vida" e "discriminação". Os resultados apoiam a viabilidade e a validade de medir diretamente as capacitações humanas para os doentes mentais e o potencial para aplicar a abordagem à mensuração de resultados.

Portanto, a Abordagem das Capacitações fornece uma terceira via de avaliação em saúde, que leva em conta não apenas os resultados na melhoria do quadro de saúde do paciente, mas também outros aspectos concernentes à sua vida, de modo que possam escolher a vida tem razão em valorizar. Ou seja, neste caso, a avaliação normalmente é feita por meio de um quadro referencial multidimensional, e não de apenas um indicador como no caso do QALY ou da Felicidade. Isso auxilia no entendimento da saúde mental, pois fornece instrumental de análise que aborda aspectos importantes da vida das pessoas que podem ser correlatos com a saúde ou não.

\section{Reflexões finais sobre a avaliação de políticas em saúde}

O objetivo deste estudo foi fazer uma revisão narrativa sobre os diferentes caminhos metodológicos para a avaliação econômica de políticas em saúde mental. Portanto, foram apresentadas três possibilidades, começando pela proposta comumente utilizada, que analisa custo-utilidade, passando pela perspectiva da felicidade, que tem crescido nas últimas décadas, e finalizando com a abordagem menos estudada empiricamente, que é a das capacitações.

Tal debate se torna relevante, pois os transtornos mentais, em especial a depressão e a ansiedade, que foram o objeto de pesquisa, constituem um fator relevante para a piora na qualidade de vida das pessoas e em níveis inferiores de bem-estar não só do indivíduo como também da sociedade. Ademais, a depressão é um caso de saúde pública, impactando em custos pessoais e sociais, reduzindo a felicidade média da população e tornando-se um fator limitante das liberdades individuais. Sendo assim, esta revisão teórica da literatura permite três considerações:

1. As pesquisas em Economia da Felicidade têm abordado a relevância da saúde para o bem-estar subjetivo dos indivíduos. Entretanto, poucos estudos tratam especificamente da saúde mental e dos problemas da depressão e da ansiedade de forma empírica. Esse é um campo próspero para estudos futuros. Ocorre que a Ciência da Felicidade tem crescido, sobretudo, nas últimas duas décadas e o tema tem recebido maior atenção de diversas áreas do conhecimento humano. Entender a influência das doenças mentais na infelicidade, comparativamente a outros fatores, pode propiciar o debate sobre as prioridades em políticas públicas. Também, pode-se analisar o custo-retorno em termos de felicidade, abordagem essa que se aproxima da Economia Tradicional.

2. Quanto à Abordagem das Capacitações, as pesquisas em saúde mental são mais restritas. Ocorre que essa é uma abordagem complexa e teoricamente desafiadora, especialmente quando se propõe a construir uma metodologia para selecionar as capacitações e analisar os resultados a partir de pesquisas aplicadas. Desse modo, mesmo em países desenvolvidos, o número de pesquisas em saúde mental tem sido limitado. Por um lado, observa-se que a Abordagem das Capacitações é um conjunto rico de princípios e ideias. Por outro lado, constata-se que as poucas pesquisas na área da saúde mental demandam estudos que superem as dificuldades teóricas, de modo a contribuir com propostas metodológicas apenas ou também por meio de resultados empíricos para determinada região.

3. Por último, considera-se que uma análise multidimensional, em que um problema é estudado de diferentes ângulos, como é o caso da avaliação de políticas públicas, permite um entendimento aprofundado e abrangente. Portanto, sugere-se que pesquisas futuras possam trabaIhar o tema da saúde mental sob o prisma de diferentes aproximações teóricas, e a Economia pode contribuir nisso proveitosamente. 


\section{Referências bibliográficas}

Anand P. Capabilities and health. J Med Ethics. 2005;31(5):299-303.

Campetti PHM, Alves TW. Economia da Felicidade: estudo empírico sobre os determinantes da felicidade em países selecionados da américa latina. Pesq Debate. 2015;26(1):99-123.

Chisholm D, Sweeny K, Sheehan P, Rasmussen B, Smit F, Cuijpers P. Scalingup treatment of depression and anxiety: a global return on investment analysis. Lancet Psychiatry. 2016;3(5):415-24.

Clark A, Flèche S, Layard R, Powdthavee N, Ward G. The key determinants of happiness and misery. In: Helliwell J, Layard R, Sachs J, eds. World Happiness Report 2017. Nova York: UN Sustainable Development Solutions Network; 2017.

Cruz CRB, Shirassu MM, Barbosa PLQ, Santana AMR. Transtornos mentais como causa de absenteísmo entre servidores públicos em São Paulo. Rev Psiquiatr Clin. 2011;38(6):265-6.

Easterlin RA. Building a better theory of well-being. In: Bruni L, Porta PL, eds. Economics \& happiness: framing the analysis. New York: Oxford University Press; 2005.

Easterlin RA. Does economic growth improve the human lot? In: David P, Reder M, eds. Nations and households in economic growth: essays in honor of Moses Abramovitz. New York: Academic Press; 1974.

Faria AC, Barboza DB, Domingos NAM. Absenteísmo por transtornos mentais na enfermagem no período de 1995 a 2004. Arq Ciênc Saúde. 2005;12(1):14-20.

Fonseca RMC, Carlotto MS. Saúde Mental e Afastamento do Trabalho em Servidores do Judiciário do Estado do Rio Grande do Sul. Psicol Pesq. 2011;5(2):117-25

Frederick S, Loewenstein G. Hedonic adaptation. In: Kahneman D, Diener E, Schwarz N, eds. Well-Being: The foundations of Hedonic psychology. Nova York: Russell Sage; 1999.

Frey BS, Stutzer A. Happiness \& Economics: how the economy and institutions affect human well-being. Princeton, New Jersey: Princeton University Press; 2002.

Goetzel R, Hawkins K, Ozminkowski RJ, Wang S. The health and productivity cost burden of the "top 10" physical and mental health conditions affecting six large U.S. employers in 1999. J Occup Environ Med. 2003:45(1):5-14.

Greenberg PE, Kessler RC, Birnbaum HG, Leong SA, Lowe SW, Berglund PA, et al. The economic burden of depression in the United States: how did it change between 1990 and 2000? J Clin Psychiatry. 2003;64(12):1465-75.

Hunt EK. História do pensamento econômico: uma perspectiva critica. $2^{\text {a }}$ ed. Rio de Janeiro: Elsevier; 2005.

Kim-Cohen J, Caspi A, Moffitt TE, Harrington H, Milne BJ, Poulton R. Prior juvenile diagnoses in adults with mental disorder: developmental follow-back of a prospective-longitudinal cohort. Arch Gen Psychiatry. 2003;60(7):709-17.

Kinghorn PB, Robinson A, Smith R. Developing the Capability Approach to Assess Quality of Life in Patients with Chronic. iHEA 2007 6th World Congress: Explorations in Health Economics Paper; 2007.

Kuklys W. A Monetary Approach to Capability Measurement of the Disabled in the UK. In: Kuklys W. Amartya Sen's Capability Approach: Theoretical Insights and Empirical Applications. Berlin, Heidelberg: Springer; 2005.

Layard R, Chisholm D, Patel V, Saxena S. Mental illness and unhappiness. In: Helliwell J, Layard R, Sachs J. World Happiness Report 2013. Nova York: UN Sustainable Development Solutions Network; 2013.

Laynard R, Clark D, Knapp M, Mayraz G. Cost-benefit analysis of psychological therapy. National Institute Economic Review. 2007;202(1):90-8.

Layard R, Clark A, Senik C. The causes of happiness and misery. In: Helliwell J, Layard R, Sachs J. World Happiness Report 2012. Nova York: UN Sustainable Development Solutions Network; 2012.
Layard R. Felicidade: lições de uma nova ciência. Rio de Janeiro: Best Seller; 2008.

Layard R. Mental health: the new frontier for labour economics. In: McDaid D, Cooper $\mathrm{CL}$, eds. Wellbeing: a complete reference guide Volume 5. The economics of wellbeing. Oxford: Wiley-Blackwell; 2014.

Lorgelly PK, Lawson KD, Fenwick EA, Briggs AH. Outcome Measurement in Economic Evaluations of Public Health Interventions: a Role for the Capability Approach? Int J Environ Res Public Health. 2010;7(5):2274-89.

Lorgelly PK, Lorimer K, Fenwick E, Briggs AH. The capability Approach: developing an instrument for evaluating public health interventions. Final Report. Glasgow: Glasgow Centre for Population Health; 2008.

Lorimer K, Lorgelly P, Fenwick E, Briggs A. Using mixed methods to operationalise the capability approach: an application in public health. 4th annual conference of the HDCA. Nova York; 2007.

Luyten J, Naci H, Knapp M. Economic evaluation of mental health interventions: an introduction to cost-utility analysis. Evid Based Ment Health. 2016;19(2):49-53.

Mcgillivray M. Human well-being: issues, concepts and measures. In: Mcgillivray M (Ed.). Human well-being: concept and measurement. New York: Palgrave Macmillan; 2007.

Mehnert T, Krauss HH, Nadler R, Boyd M. Correlates of life satisfaction in those with disabling conditions. Rehabil Psychol. 1990;35(1):3-17.

Mykletun A, Bjerkeset O, Overland S, Prince M, Dewey M, Stewart R. Levels of anxiety and depression as predictors of mortality: the HUNT study. Br J Psychiatry. 2009;195(2);118-25.

Nicholson A, Kuper H, Hemingway H. Depression as an aetiologic and prognostic factor in coronary heart disease: a meta-analysis of 6362 events among 146538 participants in 54 observational studies. Eur Heart J. 2006;27(23):2763-74.

Nussbaum MC. Creating capabilities: the human development approach. Cambridge, MA: Harvard University Press; 2011.

OECD - Organisation for Economic Co-operation and Development. Sick on the Job?: Myths and Realities about Mental Health and Work, Mental Health and Work, OECD Publishing, Paris; 2012.

Pawlina MMC, Campos AF, Ribeiro LS. Características de absenteísmo entre trabalhadores da saúde: nível central da Secretaria de Estado de Saúde/MT de 2005 a 2006. Revista Planejamento e Políticas Públicas. 2009;33:173-94.

Razzouk D. Capital mental, custos indiretos e saúde mental. In: Razzouk D, Lima MGA, Cordeiro Q (Orgs.). Saúde mental e trabalho. São Paulo: Cremesp - Conselho Regional de Medicina do Estado de São Paulo; $2016 a$.

Razzouk D. Economia da saúde aplicada à saúde mental. In: Mateus MD (Org.). Políticas de saúde mental. São Paulo: Instituto de Saúde; 2013.

Razzouk D. Por que o Brasil deveria priorizar o tratamento da depressão na alocação dos recursos da Saúde? Epidemiol Serv Saúde. 2016b;25(4)845-8.

Robeyns I. The capability approach: a theoretical survey. J Hum Develop. 2005;6(1)93-114.

Santana LL, Sarquis LMM, Brey C, Miranda FMA, Felli VEA. Absenteísmo por transtornos mentais em trabalhadores de saúde em um hospital no sul do Brasil. Rev Gaúcha Enferm. 2016;37(1):1-8.

Santos JP, Mattos AP. Absentismo-doença na prefeitura municipal de Porto Alegre, Rio Grande do Sul, Brasil. Rev Bras Saúde Ocup. 2010;35(121):148-56.

Sen AK. A ideia de justiça. São Paulo: Companhia das Letras; 2011.

Sen AK. Desenvolvimento como liberdade. São Paulo: Companhia das Letras; 2010.

Sen AK. Desigualdade reexaminada. Rio de Janeiro: Record; 2008. 
Silva EBF, Tomé LAO, Costa TJG, Santana MCCP. Transtornos mentais e comportamentais: perfil dos afastamentos de servidores públicos estaduais em Alagoas, 2009. Epidemiol Serv Saúde. 2012;21(3):505-14.

Silva Junior JS, Fischer FM. Adoecimento mental incapacitante: benefícios previdenciários no Brasil entre 2008-2011. Rev Saúde Pública. 2014;48(1):186-90.

Silva Junior JS, Fischer FM. Afastamento do trabalho por transtornos mentais e estressores psicossociais ocupacionais. Rev Bras Epidemiol. 2015;18(4):735-44.

Silva MT, Silva EM, Pereira MG. Desfechos em estudos de avaliação econômica em saúde. Epidemiol Serv Saúde. 2016;25(3):663-6.

Simon J, Anand P, Gray A, Rugkåsa J, Yeeles K, Burns T. Operationalising the capability approach for outcome measurement in mental health research. Soc Sci Med. 2013;98:187-96.
Thomas CM, Morris S. Cost of depression among adults in England in 2000. Br J Psychiatry. 2003;183:514-9.

Vos T, Flaxman AD, Naghavi M, Lozano R, Michaud C, Ezzati M, et al. Years lived with disability (YLDs) for 1160 sequelae of 289 diseases and injuries 1990-2010: a systematic analysis for the Global Burden of Disease Study 2010. Lancet. 2012;380(9859):2163-296.

WHO - World Health Organization. Depression and Other Common Mental Disorders Global Health Estimates; 2017. Available from: https://apps.who. int/iris/bitstream/handle/ 10665/254610/WHO-MSD-MER-2017.2-eng.pdf.

WHO - World Health Organization. Mental Health Atlas 2011. Geneva: World Health Organization; 2011. Available from: http://www.who.int/mental_ health/publications/mental_health_atlas_2011/en/.

Zaidi A, Burchardt T. Comparing incomes when needs differ: equivalization for the extra costs of disability in the U.K. Review of Income and Wealth. 2005;51(1):89-114. 